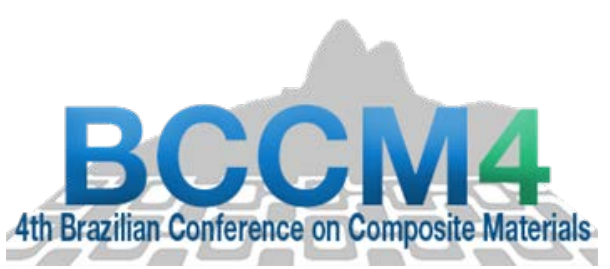

\title{
ANALYSIS OF A NOVEL INTERLOCKING ADHESIVE JOINING TECHNOLOGY FOR COMPOSITE-METAL STRUCTURES
}

\author{
Michael C. Corbett (1) and Conor T. McCarthy (1) \\ (1) Bernal Institute, School of Engineering, University of Limerick, Limerick, Ireland
}

https://doi.org/10.21452/bccm4.2018.15.01

\begin{abstract}
The incorporation of composite materials in the future's lightweight vehicle structures depends on the availability of suitable joining techniques. A novel joining concept is presented herein. It employs interlocking morphology formed on the surfaces of composite (female) and metal (male) adherends that are coupled with a layer of adhesive to interlock in shear. This acts to increase load transfer in the central region of the joint overlap. In the present work, the concept is investigated with a sophisticated finite element model, incorporating: adhesive damage through a cohesive zone model (CZM), composite intralaminar damage through a continuum damage model, composite interlaminar damage through a CZM, and metallic damage through Abaqus' ductile damage model. The concept demonstrates impressive improvements in performance compared to a standard adhesive joint. Its maximum applied load is increased by 33.7\%, while the work to failure is $582.9 \%$ greater than the standard joint. These improvements are achieved as the interlocking geometry prevents the propagation of damage in the adhesive and damage develops in the composite adherend prior to failure.
\end{abstract}

Keywords: joining, continuum damage model, cohesive zone model, finite element analysis

\section{INTRODUCTION}

The demand to minimise the environmental impact of the transportation industry is motivating the lightweighting of vehicles. Research shows that the future's lightweight vehicle structures will be multi-material constructions, incorporating high-strength metallic alloys and composite materials. Adhesive bonding is a primary technique for the joining of composite to metal. However, it presents a number of inherent weaknesses that have restricted its uptake in load bearing applications. This research considers a novel, hybrid, mechanical-adhesive technique for joining materials. The technique employs interlocking bond-surface morphology formed on the surfaces of male (metallic) and female (composite) adherends that are coupled with a layer of adhesive to mechanically interlock in shear. The interlocking surfaces serve to activate the central overlap region of the adhesively bonded joint, which is known to typically be relatively inactive for load transfer in comparison to the overlap ends. Thus, the weight penalty and stress concentration associated with mechanical fastening are negated, while an additional, mechanical 
loading mechanism is presented in the adhesive joint to improve durability. The concept has previously been investigated for a metal-metal joining configuration [1] and shown compelling improvements in joint strength and toughness. Herein, the concept is investigated, with finite element analysis, in a multi-material, composite-metal joining configuration. Detailed dimensions of the joint geometry are not provided herein and the performance attributes of the concept are non-dimesionalised with respect to a standard adhesive joint, modelled with equivalent material properties, to protect the nobility of the concept. It was also necessary to hide the interlocking geometry in the images presented herein in order to protect the intellectual property of the authors at the present time.

\section{JOINT SPECIFICATION AND MATERIALS}

The single-lap joint (SLJ) is one of the most commonly occurring joining configurations and is that most often used for testing adhesives and for characterising mechanically fastened joints. It is therefore an effective starting point to investigate the efficacy of a new, hybrid joining concept. The joint geometry, shown in Figure 1, was selected in consideration of the American Society for Testing and Materials (ASTM) standards: ASTM D 1002 and ASTM D 5868. The joint was loaded in quasi-static, displacement controlled tension, as shown in Figure 1.

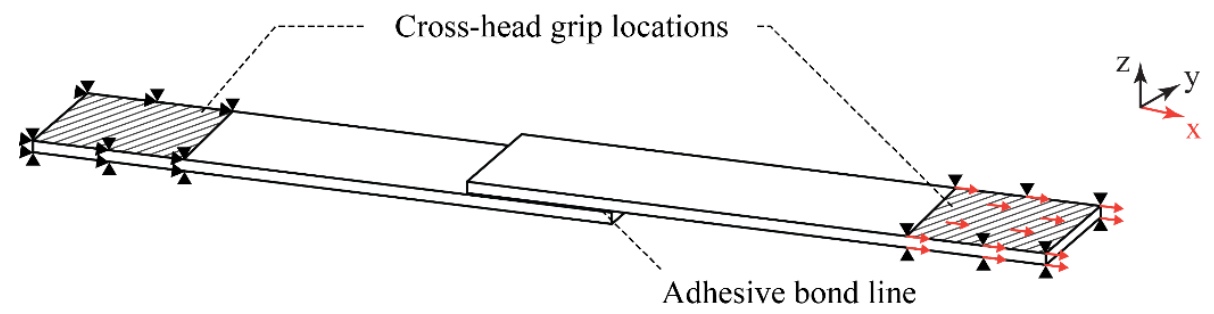

Figure 1: Single lap joint geometry and boundary conditions.

The interlocking adaptation of the SLJ is comprised of male and female adherends, as shown in Figure 2, which had interlocking geometry formed on their surfaces. The female adherend, which was a laminated composite material, HTA/6376, was characterised by depressions in its surface corresponding to the interlocking morphology. A symmetric, quasi-isotropic laminate stacking sequence was employed, [45/0/-45/90]2s. The male adherend, which was an aluminium alloy, AA5754-H111, was distinguished by protruding profiles which are defined to fit the female adherend with a constant clearance to accommodate the adhesive, which was an epoxy resin from Nagase ChemteX, XNR6823 [2].

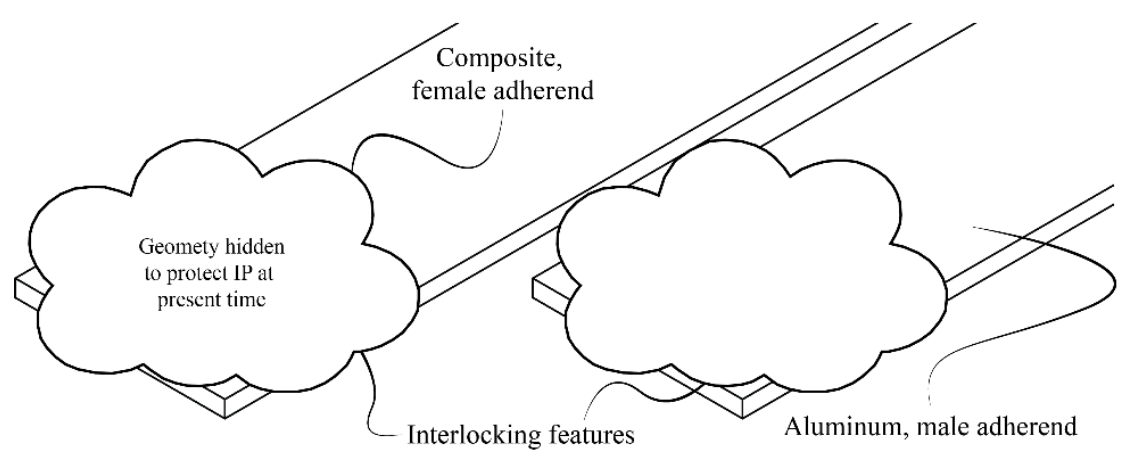

Figure 2: Male and female adherends, with interlocking morphology dimensions. 


\section{FINITE ELEMENT JOINT MODEL}

A three-dimensional finite element (FE) model of the joint was developed in Abaqus ${ }^{\circledR}$. It incorporated separate parts for each of the male adherend, the female adherend, and the adhesive. Partitioning was applied to each part to facilitate structured meshing with solid hexahedral elements. Layered partitioning was applied to the composite adherend and each ply was discretised by a single layer of elements, which were orientated according to the laminate stacking sequence. First-order, reduced integration elements (C3D8R in Abaqus ${ }^{\circledR}$ ) with hourglass control were used to discretise the adherends, and the adhesive in models that considered its elastic response (six elements through thickness). In models considering adhesive damage it was discretised by a single layer of 8-node cohesive elements (COH3D8 in Abaqus ${ }^{\circledR}$ ). ${ }^{\circledR}$ ).

\subsection{Material Models}

The mechanical response of the aluminium alloy, AA5754-H111 was characterised by elasticplastic behaviour, incorporating von Mises yield criterion and isotropic strain hardening defined through Swift's law:

$$
\sigma=A\left(\varepsilon_{o}+\varepsilon_{p}\right)^{\prime \prime}
$$

Eq. 1

where $\sigma$ is the true stress, $A$ is a strength coefficient, $\varepsilon_{o}$ is the true strain the onset of yielding, $\varepsilon_{p}$ is the true plastic strain, and $n$ is the strain hardening exponent. The stress-strain response was produced from experiments; salient properties are summarised in Table 1. Damage initiation in the metal was considered through the ductile criterion in Abaqus ${ }^{\circledR}$ [3]. This is a phenomenological model for predicting damage onset as a function of stress triaxiality and the third invariant of deviatoric stress, which is related to the Lode angle [3]. The experimental data for this criteria was obtained from literature [4], which shows that ductile fracture of AA5754 shows significant dependence on these parameters. Linear damage evolution based on effective plastic displacement was applied to approximate the behaviour observed during experiments.

In models considering the stress distribution in the adhesive prior to the onset of damage, the adhesive was considered an elastic material. Subsequently, to determine the influence of damage and fracture on performance of the joint, the adhesive was represented by the uncoupled, mixedmode cohesive zone model (CZM) in Abaqus ${ }^{\circledR}$ [3], which simplifies the macroscopic response of the adhesive layer into a bi-linear traction-separation law. The elastic response of the cohesive law was governed by the elastic properties of the adhesive, equal to the Young's modulus, $E$ in the normal direction, and the shear modulus, $G$ in shear [2]. The quadratic stress criterion was used to determine a damage initiation,

$$
\left(\frac{\left\langle t_{n}\right\rangle}{t_{n}^{o}}\right)^{2}+\left(\frac{t_{s}}{t_{s}^{o}}\right)^{2}+\left(\frac{t_{t}}{t_{t}^{o}}\right)^{2}=1
$$

where $t_{n}, t_{s}$ and $t_{t}$, are tractions in the normal, and first and second shear directions, respectively, and $t_{n}^{o}, t_{s}^{o}$ and $t_{t}^{o}$ are critical traction values for damage initiation in each mode. Once damage initiates, the stiffness of the cohesive elements softens progressively according to a scalar damage parameter, which evolve monotonically from zero to one upon further loading. The evolution of damage is controlled by the linear energetic criterion,

$$
\left(\frac{G_{n}}{G_{n}^{c}}\right)^{\alpha}+\left(\frac{G_{s}}{G_{s}^{c}}\right)^{\alpha}+\left(\frac{G_{t}}{G_{t}^{c}}\right)^{\alpha}=1
$$


where $G_{n}, G_{s}$, and $G_{t}$ are the normal and tangential fracture energy release rates, respectively, $G_{n}^{c}$, $G_{s}^{c}$, and $G_{t}^{c}$ are critical fracture energies in each modes, and $\alpha=1$ for the linear criterion. Once this criterion is satisfied, the damage parameter is set to 1 , the element may no longer carry load and is deleted from the model, allowing fracture to propagate. The properties of the adhesive were taken from [2] and are provided in Table 2.

Table 1: Mechanical properties of AA5754-H111.

\begin{tabular}{ccccc}
\hline$E$ & $v$ & $\sigma_{y}$ & $A$ & $n$ \\
\hline $68 \mathrm{MPa}$ & 0.33 & $90 \mathrm{MPa}$ & $371.72 \mathrm{MPa}$ & 0.2085 \\
\hline
\end{tabular}

Table 2: Mechanical properties of XNR6823 [2].

\begin{tabular}{cccccc}
\hline$E$ & $G$ & $t_{n}^{o}$ & $t_{s}^{o}$ & $G_{n}^{c}$ & $G_{s}^{c}$ \\
\hline $2600 \mathrm{MPa}$ & $1000 \mathrm{MPa}$ & $57 \mathrm{MPa}$ & $32.9 \mathrm{MPa}$ & $1.18 \mathrm{~N} / \mathrm{mm}$ & $1.5 \mathrm{~N} / \mathrm{mm}$ \\
\hline
\end{tabular}

The constitutive behaviour of the composite material, HTA/6376, was represented by an intralaminar damage model, developed by [5], implemented through a VUMAT. It includes in-plane non-linear shear and features an innovative treatment of load reversals. The maximum stress criteria is employed for the prediction of tensile and compressive fibre failures. Puck's criteria is incorporated in order to predict tensile and compressive intralaminar matrix failure by checking for damage initiation on multiple potential fracture planes. Subsequent to initiation, damage evolves irreversibly through a non-linear softening law and the crack band model is used to mitigate mesh sensitivity by adjusting the final failure strain of each element based on its characteristic length and fracture energy. Once an element has been fully degraded it is subject to deletion based on a shear criterion. Experiments were previously conducted by O'Higgins [6] to determine the material properties and describe the damage development laws of HTA/6376, relevant damage model properties are outlined in Table 3. To account for interlaminar damage, zero-thickness layers of cohesive elements were included at each ply boundary. The uncoupled, mixed-mode CZM in Abaqus ${ }^{\circledR}$ [3] defined the mechanical behaviour of these elements through a bi-linear traction-separation law with linear softening. The initial stiffness of the cohesive elements was governed by a penalty parameter, $K$. The initiation of damage was determined by the quadratic stress damage initiation criterion (Eq. 2), while damage evolution was controlled by the linear fracture energetic criterion (Eq. 3). The CZM properties, corresponding to [7], are provided in Table 4.

Table 3: Damage model properties for HTA/6376 [6].

\begin{tabular}{cccccccc}
\hline$X_{T}$ & $X_{C}$ & $Y_{T}$ & $S_{12}$ & $S_{n t}$ & $\mu_{t}$ & $\mu_{l}$ & $G^{f}$ \\
\hline $2170 \mathrm{MPa}$ & $1600 \mathrm{MPa}$ & $73.3 \mathrm{MPa}$ & $82.6 \mathrm{MPa}$ & $94.2 \mathrm{MPa}$ & 0.29 & 0.37 & $40 \mathrm{~N} / \mathrm{mm}$ \\
\hline
\end{tabular}

Table 4: Cohesive zone model properties for delamination of HTA/6376 [7].

\begin{tabular}{ccccc}
\hline$K$ & $t_{n}^{o}$ & $t_{s}^{o}$ & $G_{n}^{c}$ & $G_{s}^{c}$ \\
\hline $1 e^{5} \mathrm{~N} / \mathrm{mm}^{3}$ & $30 \mathrm{MPa}$ & $58.89 \mathrm{MPa}$ & $0.26 \mathrm{~N} / \mathrm{mm}$ & $1.002 \mathrm{~N} / \mathrm{mm}$ \\
\hline
\end{tabular}




\section{RESULTS AND DISCUSSION}

\subsection{Elastic stress distribution}

The elastic stress distribution in the adhesive of the interlocking joint was examined by applying a constant load corresponding to linear deformation of the joint. The resulting distributions of normal and tangential stress are shown in Figure 3, and compared to a standard adhesive joint in Figure 4. To discuss the influence of the interlocking morphology on the stress distribution, the joint overlap is described by its female end, i.e. the end of the overlap closest to the constraint of the female adherend $(\mathrm{x}=0)$ and vice versa, its male end $(\mathrm{x}=1)$.
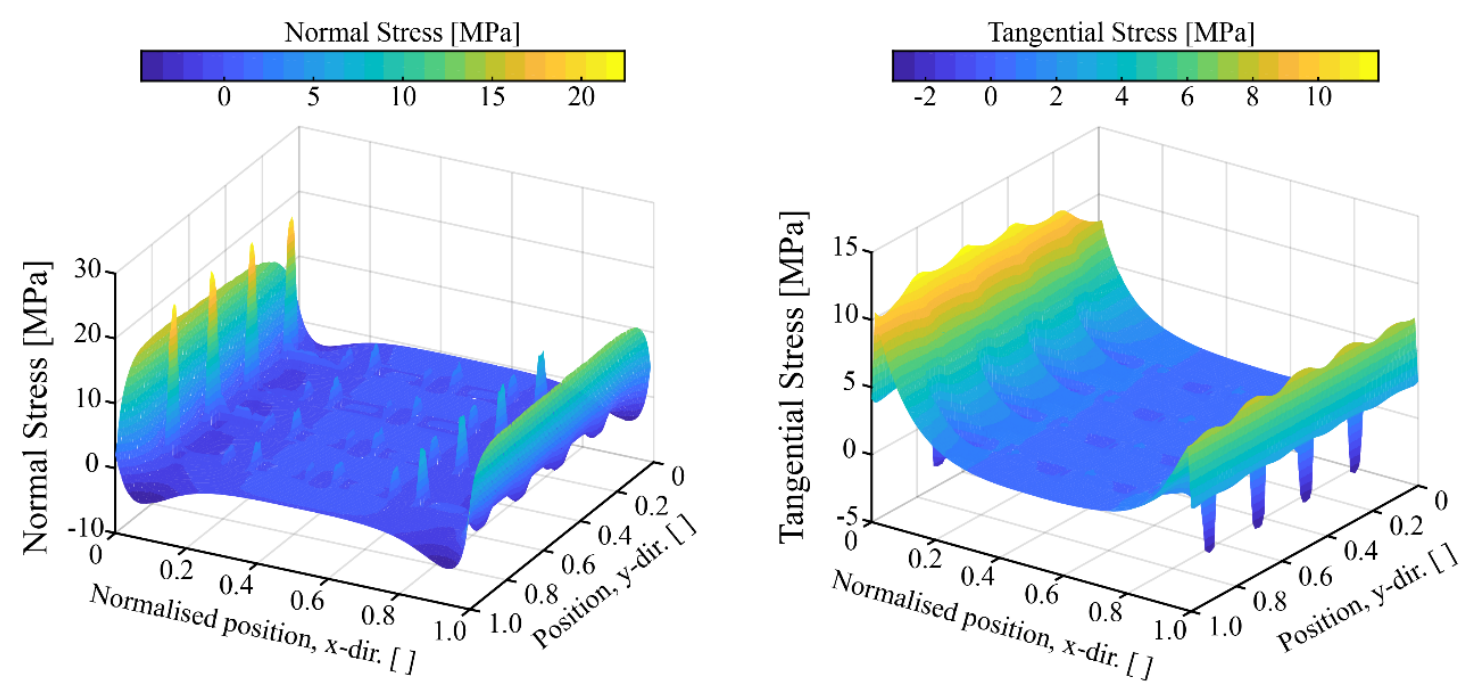

Figure 3: Normal (left) and tangential (right) stress in the adhesive of the interlocking joint.
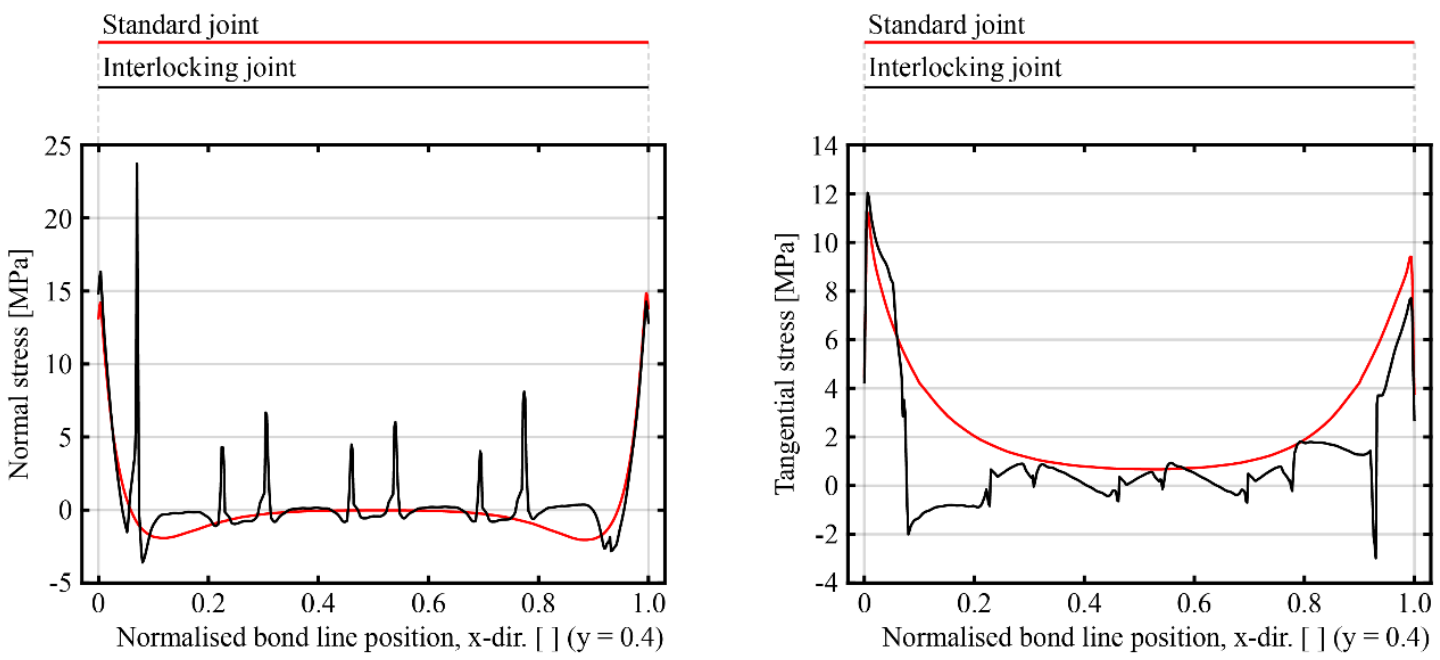

Figure 4: Normal (left) and tangential (right) stress in a section (at $y=0.4$ ) of the adhesive of the interlocking joint compared to a standard joint.

The normal and tangential distributions of stress in the adhesive at the ends of the interlocking joint overlap correspond closely that observed in the standard joint. Peaks in tensile normal stress at the female and male ends of the interlocking joint are slightly higher and lower, respectively, 
than the standard joint. Concentrations in tensile normal stress also exist acting on the interlocking features (x-dir). Similarly, peaks in tangential stress at the female and male ends of the joint are slightly higher and lower, respectively, than the standard joint. This indicates that damage may initiate at the female end of the overlap. Minimal tangential stress is observed in the adhesive in the central part of the overlap of the interlocking joint.

\subsection{Progressive damage and failure}

The mechanical response of the interlocking adhesive joint is compared to that of a standard adhesive joint (without interlocking geometry), which has been modelled with equivalent material properties, in Figure 5; important performance characteristics are summarised in Table 5. The interlocking joint achieves a significantly greater displacement than the standard joint prior to failure. Accordingly, the maximum applied load of the interlocking joint is $33.7 \%$ greater than that of the standard joint, and more remarkably, the work required to fracture the interlocking joint is $582.9 \%$ greater. The response curves exhibit highly nonlinear response, with decreasing stiffness as the joint extension increases. This is most apparent for the interlocking joint and is indicative of plastic deformation of the aluminium adherend.

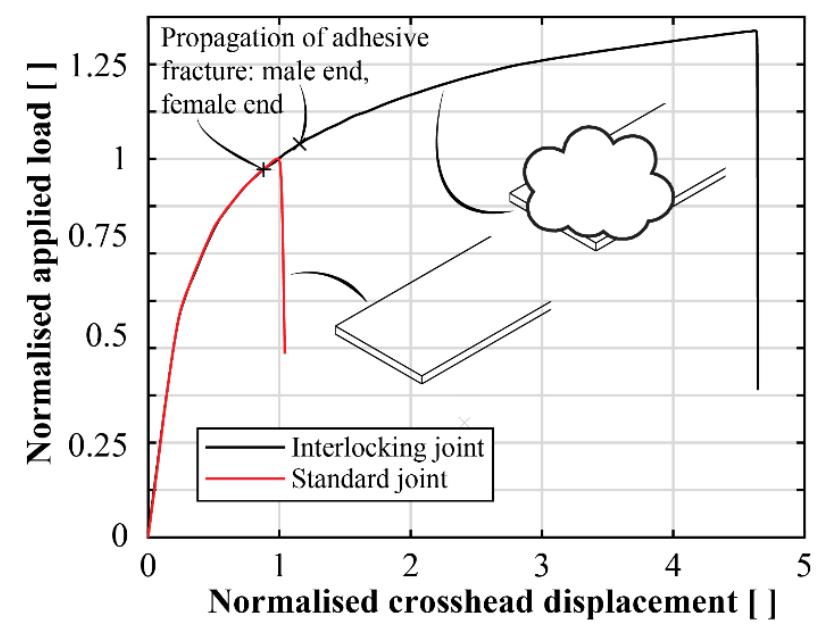

Figure 5: Mechanical response of the interlocking joint compared to a standard joint.

Fracture initiated and began to propagate almost simultaneously from both ends of the standard joint's overlap at the point of its maximum load; after which, fracture propagated rapidly through the remaining adhesive, resulting in failure. In contrast to this, the points at which the fracture began to propagate in the adhesive from each of the female and male ends of the overlap of the interlocking joint are indicated in Figure 5. Fracture initiated in the adhesive at the female end of the interlocking joint, corresponding to the increased adhesive stress discussed in Section 4.1. It subsequently initiated in the adhesive at the male end of the overlap. However, the interlocking geometry prevented adhesive fracture from propagating across the remaining adhesive by restricting the relative displacement of the adherends as load was transferred mechanically through the interlocking features. The progress of damage in the adhesive is illustrated in Figure 6. A delamination also initiated at the female end of the overlap following the initiation of adhesive fracture, as shown in Figure 7a. 
Table 5: Normalised performance of the interlocking adhesive joint.

\begin{tabular}{l|ccc}
\hline & Interlocking joint & Standard joint & Difference \\
\hline Max. applied load & 1.337 & 1 & $+33.7 \%$ \\
Work to failure & 6.829 & 1 & $+582.9 \%$ \\
\hline
\end{tabular}

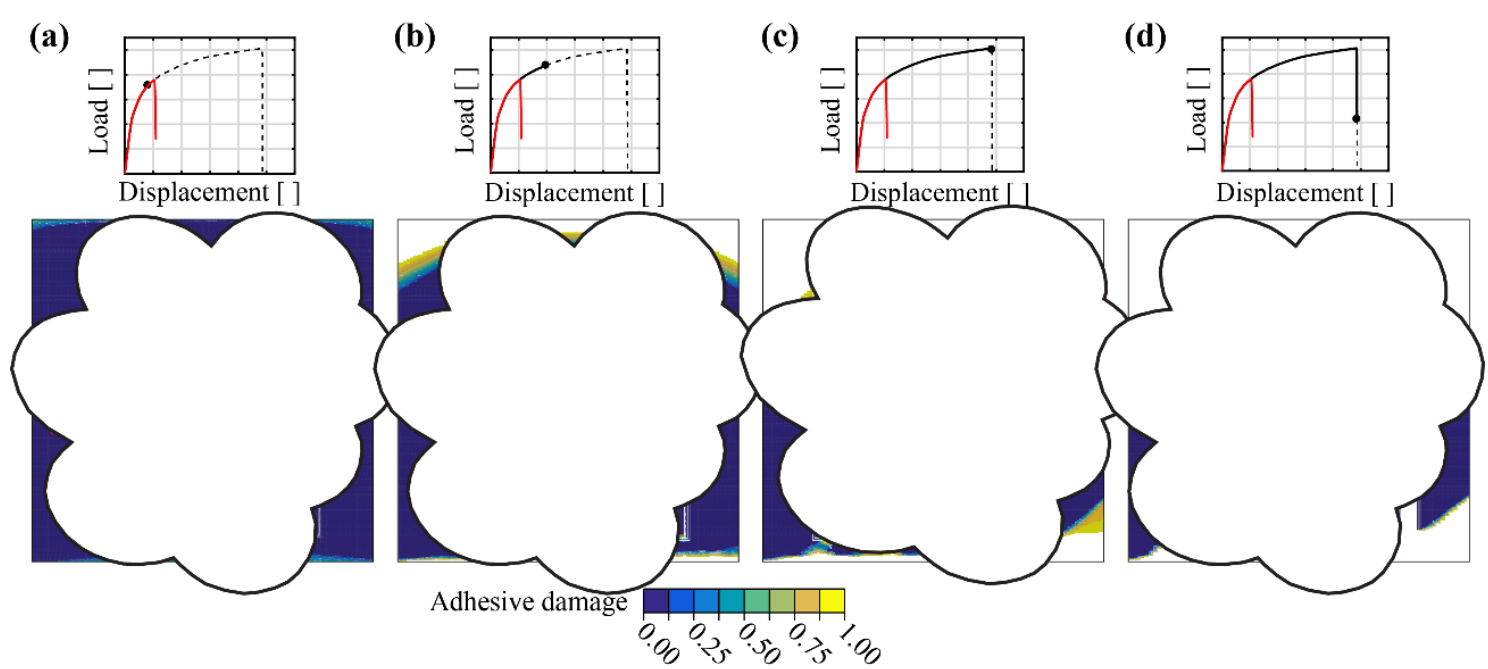

Figure 6: Damage in the adhesive of the interlocking joint at four points in the load history.

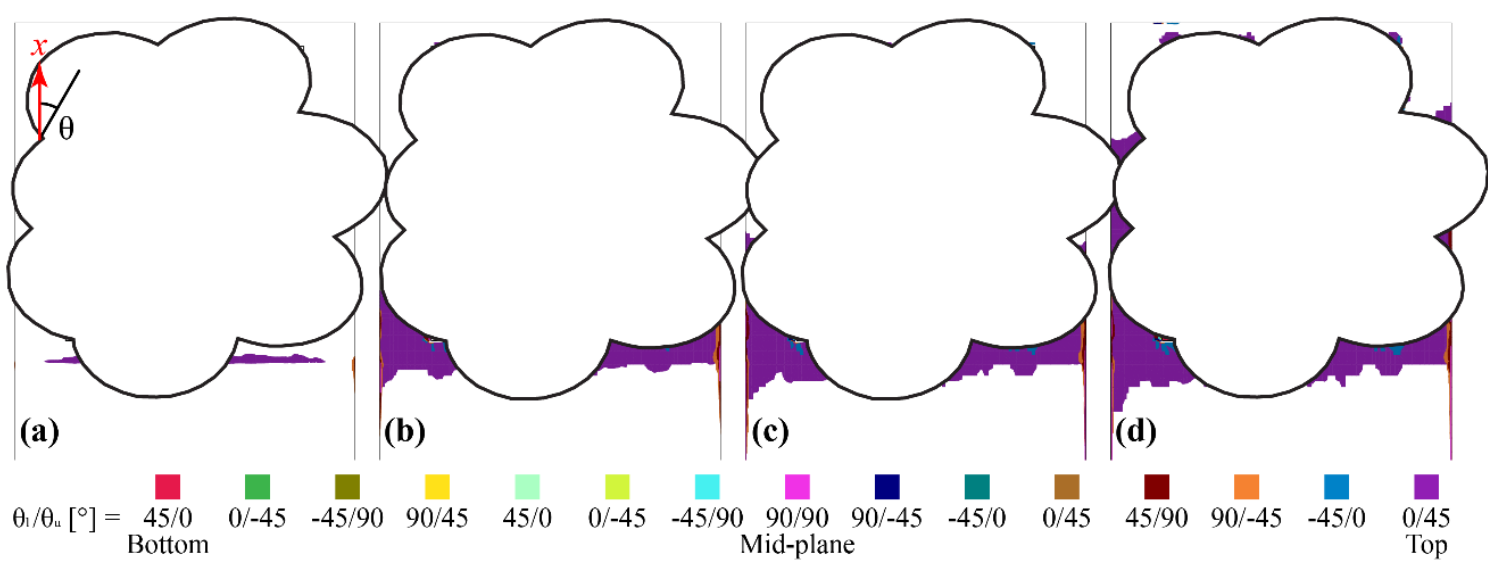

Figure 7: Delamination in the composite, female adhered of the interlocking joint at four points in the loading history (points correspond to a-d in Figure 6).

The interlocking joint subsequently sustained increasing load as damage developed in the adhesive and in the composite adherend. Compressive stress was induced on the adhesive at the male end of each interlocking profile as load was transferred mechanically through these features. Minor delaminations developed around the profiles closest to the female end of the overlap, while the large delamination propagated between the two plies closest to the joint's bonding plane at the female end of the overlap (Figure $7 \mathrm{~b}, \mathrm{c}$ ). Failure of the joint was ultimately a result of this 
delamination propagating across the overlap region while some adhesive remained in-tact, adhering the top ply of the composite to the male adherend.

\section{CONCLUSIONS}

The interlocking adhesive joint demonstrates significant increases in performance compared to a standard adhesive joint, with a 33.7\% improvement in the maximum applied load and 582.9\% in work to failure. These improvements are achieved as the interlocking morphology of the joint prevents the propagation of adhesive fracture across the central region of the joint overlap. Once fracture initiates at the ends of the joint overlap, the interlocking geometry assumes load transfer mechanically across the joint interface. This prevents relative displacement of the adherends which prevents further damage to the adhesive. Thus, significant plastic deformation occurs in the metal and damage develops in the composite prior to failure, which ultimately occurred through delamination. Importantly, this presents a shift in failure mode from adhesive/cohesive failure observed in the standard adhesive joint, while the additional deformation mechanisms engaged in the interlocking joint are central to increasing the work required to fracture the joint. The developments suggest that the interlocking adhesive joint would find suitable applications in automotive crash structures of lightweight, composite-metal vehicles, where the work to failure is a critical performance criterion.

\section{ACKNOWLEDGEMENTS}

The authors acknowledge the financial support of Science Foundation Ireland under Grant No. 13/IA/1833 and the Irish Research Council (IRC) for funding under their Government of Ireland Postgraduate Scheme through Grant No. GOIPG/2014/945. The authors also wish to acknowledge the DJEI/DES/SFI/HEA Irish Centre for High-End Computing (ICHEC) for the provision of computational facilities and support.

\section{REFERENCES}

[1] M. C. Corbett, P. A. Sharos, M. Hardiman, and C. T. McCarthy, "Numerical design and multiobjective optimisation of novel adhesively bonded joints employing interlocking surface morphology,” Int. J. Adhes. Adhes., 78, pp. 111-120, (2017).

[2] T. E. A. Ribeiro, R. D. S. G. Campilho, L. F. M. da Silva, and L. Goglio, "Damage analysis of composite-aluminium adhesively-bonded single-lap joints,” Compos. Struct., 136, pp. 2533, (2016).

[3] Abaqus, “User’s Manuals, Version 6.14,” Providence, RI, USA, (2014).

[4] M. R. Millán, “Análisis experimental y numérico de la influencia del estado tensional en la deformación de fallo de elementos estructurales de aluminio,” Charles III University of Madrid, (2013).

[5] B. Egan, M. A. McCarthy, R. M. Frizzell, P. J. Gray, and C. T. McCarthy, "Modelling bearing failure in countersunk composite joints under quasi-static loading using 3D explicit finite element analysis,” Compos. Struct., 108, no. 1, pp. 963-977, (2014).

[6] R. M. O’Higgins, “An Experimental and Numerical Study of Damage Initiation and Growth in High Strength Glass and Carbon Fibre-Reinforced Composite Materials,” University of Limerick, (2007).

[7] C. Wang and $\mathrm{X} . \mathrm{Xu}$, "Cohesive element analysis of fatigue delamination propagation in composite materials with improved crack tip tracking algorism,” Compos. Struct., 134, pp. 176-184, (2015). 